\title{
Miranda
}

Revue pluridisciplinaire du monde anglophone /

Multidisciplinary peer-reviewed journal on the English-

speaking world

5 | 2011

South and Race / Staging Mobility in the United States

\section{Le héros de la Frontière, un mythe de la fondation en mouvement}

Daniel Agacinski

\section{OpenEdition \\ Journals}

Édition électronique

URL : http://journals.openedition.org/miranda/2403

DOI : 10.4000/miranda.2403

ISSN : 2108-6559

Éditeur

Université Toulouse - Jean Jaurès

\section{Référence électronique}

Daniel Agacinski, «Le héros de la Frontière, un mythe de la fondation en mouvement », Miranda [En

ligne], 5 | 2011, mis en ligne le 29 novembre 2011, consulté le 16 février 2021. URL : http:// journals.openedition.org/miranda/2403; DOI : https://doi.org/10.4000/miranda.2403

Ce document a été généré automatiquement le 16 février 2021.

\section{cc) (i) () $\Theta$}

Miranda is licensed under a Creative Commons Attribution-NonCommercial-NoDerivatives 4.0 International License. 


\title{
Le héros de la Frontière, un mythe de la fondation en mouvement
}

\author{
Daniel Agacinski
}

1 L'Ouest des États-Unis et la «Frontière » peuvent être vus à la fois comme des réalités historiques, géographiques et économiques déterminées et comme des interprétations de ces réalités, c'est-à-dire comme des concepts forgés pour leur donner une unité et une signification. Ces concepts interprétatifs n'ont pas d'abord été élaborés en tant qu'outils théoriques; ils semblent émaner de l'environnement même qu'ils désignent, et ont été repris, aussi bien par les hommes de l'Ouest que par ceux de l'Est, pour définir un espace et un mode de vie spécifiques. On peut alors les appeler des mythes, au sens où le concept de mythe, en général, désigne une représentation imaginaire et populaire qui se rapporte à un élément important de notre existence, comme notre origine, notre mode de vie, notre rapport à la nature, notre place dans le monde et dans l'histoire. L'Ouest et la Frontière auraient ainsi une existence double : d'une part une existence spatiale et historique, car la frontière entre les territoires colonisés et les terres en friche a bel et bien existé quelque part dans l'espace concret et dans l'histoire du continent nord-américain ; et de l'autre une existence psychique, en tant qu'espace mythique, c'est-à-dire en tant que représentation imaginaire d'un espace qui est davantage déterminé par le sens qu'on lui prête que par son emplacement géographique précis.

\section{Une mythologie narrative et située}

2 Prise en elle-même, cette idée n'est guère originale : l'Ouest américain est en effet de nos jours perçu d'abord comme un espace imaginaire ou fantasmé, et les processus de sa mythification ont déjà été étudiés. Il convient cependant de se demander, plus particulièrement, à quelle espèce de mythe on a affaire lorsqu'on parle de la Frontière. Les mythes de la Frontière ont été transmis par des supports qui, s'ils sont très variés, sont tous caractérisés par la très large diffusion qu'ils assurent : la presse de brochure et les dime novels, les chansons, les illustrations consacrées à l'Ouest et les films 
hollywoodiens du genre "western » ont partagé le même succès populaire. Sur chacun de ces supports, la Frontière a été le point de départ de récits - de récits présentant des personnages singuliers, ayant existé et agi d'une manière décisive pour la communauté qui les entourait. En tant qu'ils sont faits de récits d'action généralement associés à des noms propres, de lieux et de personnages, en tant qu'ils circulent et forment une série de traditions à la fois orales, écrites et picturales, on peut dire que les mythes de l'Ouest prennent la forme spécifique de la légende. La légende est précisément ce type de mythe qui donne une signification générale à un épisode historique réel particulier, et qui donne à ses personnages le statut de héros, c'est-à-dire à la fois d'hommes représentatifs d'un lieu et d'un temps, et d'acteurs décisifs dans une histoire qui les dépasse. Ceci est manifeste dans le cas des légendes de l'Ouest, qui prennent systématiquement appui sur tel ou tel événement, daté et situé, tiré de la vie de la Frontière, et le transforment en une véritable geste que l'on s'empresse de chanter et de diffuser au gré des itinérances des musiciens, des journalistes, des romanciers, ou simplement des hommes qui aiment raconter des histoires.

3 L'intérêt, en l'occurrence, de ce concept de légende par rapport à celui, plus générique, de mythe, réside dans sa dimension plus explicitement narrative que symbolique. L'anthropologie nous a donné l'habitude de lire les mythes comme des structures reposant principalement sur l'usage de la métaphore, et ce type d'analyse a constitué un moment nécessaire de l'étude des mythologies, permettant notamment d'en dégager les parentés fondamentales, quelles que soient les aires culturelles dans lesquelles elles apparaissent ou les arts qui les transmettent. Cependant, il nous semble que la mythologie de l'Ouest américain doit être située pour être comprise, c'est-à-dire qu'il faut la regarder comme étant à la fois produite dans un contexte historique et politique donné, au terme de procédures matérielles particulières, et comme étant dans son contenu même relative à une situation déterminée dans le temps et dans l'espace : la conquête puis la colonisation des terres vierges de l'Ouest de l'Amérique du Nord. Le sens des légendes de l'Ouest étant lui-même un sens spatial (l'investissement imaginaire d'un espace concret et réel), il serait sans doute insuffisant de les lire du seul point de vue de leur structure, en y recherchant uniquement l'universel humain dont elles seraient la traduction métaphorique: l'inscription du mythe dans une conjoncture historique semble prendre ici une tout autre importance que dans le cas de mythes comme celui de Sisyphe, par exemple.

4 Certes, l'interprétation des mythes en termes de symboles ou de signes n'est pas nécessairement incompatible avec un effort consistant à en situer historiquement la genèse: Barthes, par exemple, lit les mythes de son temps avec les catégories de la sémiologie et avec celles de l'idéologie. Cependant, l'idée de signe conduit à regarder le mythe comme un système, comme une totalité dont le sens est donné en bloc; et c'est une telle approche qui fait dire à Barthes du mythe qu'il est « une image naturelle »du réel historique, « une parole dépolitisée », constituée " par la déperdition de la qualité historique des choses » (216-217).

5 Le concept de légende, tel que nous voudrions l'utiliser, en tant qu'il implique directement l'idée de narrativité, permet quant à lui de lire autrement certains dispositifs mythologiques, de voir qu'ils ne constituent pas nécessairement une dépolitisation anhistorique des représentations, et qu'ils peuvent au contraire donner les clés d'une lecture politique et historique des réalités qu'ils évoquent. Le paradigme du mythe comme signe global ne pouvait pas faire droit à la pluralité, à l'équivocité qui 
est à l'œuvre dans le récit légendaire, comme dans tout récit, dès lors qu'il met en jeu plusieurs acteurs, plusieurs perspectives ; or l'identification d'une pluralité d'instances constitue le premier moment de la lecture politique d'une situation donnée. Ainsi, parler de légende nous semble nécessaire pour comprendre le type de rapport au temps, à l'espace et à la politique qui est instauré par les récits populaires des actions des hommes de la Frontière.

Qu'est-ce qui permet pour autant de faire de cette tradition légendaire un élément décisif de la représentation que l'Amérique se fait d'elle-même, une clé de la mise en scène de son identité ? Non seulement les récits des hauts faits de la Frontière ont circulé massivement sous toutes leurs formes pendant plus d'un siècle, mais ils jouent surtout un rôle dans la façon dont les États-Unis se racontent leur propre histoire politique; plus qu'un simple épisode de la vie du pays, la conquête de l'Ouest représente une scène fondatrice, maintes fois répétée le long de la Frontière. Dompter les contraintes naturelles, vaincre les Indiens, discipliner les cowboys et les hors-la-loi : tels sont les trois actes par lesquels la société américaine s'établit sur l'ensemble de son territoire, et telle est la matière inépuisable des chansons, des romans et des films. Dès lors, il semble possible de considérer ces légendes de la Frontière comme l'épopée nationale propre des Etats-Unis, dans la mesure où l'œuvre épique est précisément celle qui repose sur un récit relatant les actions qui ont contribué à la fondation de la communauté. Et ce qui caractérise l'épopée, si l'on suit l'analyse qu'en fait Hegel dans l'Esthétique, c'est son caractère doublement politique: d'une part elle se joue dans un monde où le droit est encore imparfaitement établi, dans lequel il revient à des individus d'instaurer, héroïquement, un véritable ordre civil ; d'autre part, elle est faite de «l'ensemble de la conception du monde et de la vie d'une nation, présentée sous la forme objective d'événements réels » (Hegel 1979, 101). Elle est donc poésie politique en tant que poésie nationale, c'est-à-dire qu'elle traduit dans un récit la façon dont un peuple déterminé se représente la signification de son existence politique et se raconte sa venue à l'être en tant que peuple.

7 Dans la mesure où les légendes de l'Ouest correspondent à ces critères, on peut reprendre la formule de Robert Pippin, qui, lui-même, à la suite de Kurt Bayertz, trouve chez Hegel les concepts qui lui permettent de penser la dimension proprement épique de ces histoires: "Les Grecs ont l'Iliade; les Juifs, la Bible hébraïque; les Romains, l'Énéide; les Allemands, le Niebelungenlied; les Scandinaves, la saga de Njáll; les Espagnols, le Cid ; les Anglais ont les légendes arthuriennes. Les Américains ont John Ford $»^{1}$. En deçà même du cinéma de Ford, c'est à partir de l'expérience de la Frontière, qui est l'expérience la plus proprement américaine, la plus étrangère aux nations européennes, que se construit la légende singulière du peuple américain. ${ }^{2}$

\section{Paradoxes de la mobilité des héros et de la Frontière}

8 Une fois la tradition des légendes de l'Ouest comprise comme la matrice d'un épos national américain, il faut encore analyser le contenu de ces histoires pour en comprendre les effets sur la représentation que l'Amérique se fait d'elle-même à travers elles. L'un des caractères essentiels de cette tradition est, par sa définition même, son articulation à l'espace : non seulement ces légendes sont situées dans un espace déterminé, mais surtout elles instaurent, par le type d'action qu'elles 
représentent, un certain rapport à l'espace, marqué par le motif de l'itinérance, de la mobilité. Des premiers aux derniers héros de l'Ouest - des pionniers aux hommes de loi -, tous sont des personnages en mouvement, de friche en friche, de ville en ville, de territoire en territoire. La mobilité n'est pas pour eux une propriété accidentelle : leur œuvre ne peut s'accomplir que s'ils sont mobiles, et leurs attributs les plus visibles (le plus souvent, un cheval) les identifient comme itinérants. S'ils vivent sur la Frontière, ce n'est pas sur le mode d'une station fixe, mais ils la parcourent et la modifient; ce sont des agents itinérants qui contribuent de manière décisive, selon le mythe, à la transformation du monde de la Frontière. Les défricheurs comme Daniel Boone ou Davy Crockett transforment une forêt en terre cultivable; les héros du Railroad métamorphosent les conditions de la mobilité sur la Frontière; et les fines gâchettes au service de la loi, comme Pat Garett ou Wyatt Earp, pacifient les villes de l'Ouest. Si bien que, chacun dans son genre, ces héros itinérants déplacent eux-mêmes la Frontière par leur action. Plus précisément, ils changent le statut du territoire qu'ils ont parcouru : la terre conquise par un groupe pionnier n'est plus une friche; la ville pacifiée par un marshal exceptionnel n'est plus une zone de non-droit. Autrement dit, c'est le régime spatial propre à la Frontière qui, en tant qu'il produit une instabilité et une violence permanentes, rend nécessaire l'intervention de tels personnages héroïques, dont l'action, ponctuelle, va consister à résoudre une crise typique de la vie de l'Ouest. Ils ne peuvent agir qu'une seule fois en chaque lieu, et tel est d'ailleurs le motif même de leur itinérance : une fois un terrain défriché, il faut aller en découvrir un autre; une fois une ville pacifiée, Wyatt Earp est appelé dans le comté voisin. Ce qui donne une telle importance à la mobilité du héros de l'Ouest, c'est qu'elle contribue à la puissance du mythe : comme ses hauts faits sont chantés dès qu'ils sont accomplis, et comme leur récit parcourt la Frontière, le héros itinérant est toujours précédé, où qu'il aille, par sa propre légende, diffusée d'abord par la presse populaire et les chansons, ce qui renforce son prestige et son efficacité ${ }^{3}$.

D'autre part, l'errance du héros rend compte du tragique de sa condition car, par son action même, il abolit ses propres conditions de vie: par exemple, sitôt les bandits cowboys chassés ou punis, sitôt la loi et l'ordre instaurés dans une ville, le héros qui a permis cette instauration devient superflu et même dangereux. Ainsi, le double mouvement ( $\mathrm{du}$ héros et de la Frontière) est paradoxal dans la mesure où sa signification réside dans sa fin, dans son terme, qui est, sinon l'immobilité, du moins l'installation sédentaire, la colonisation de l'espace et son occupation par des habitants fixes : c'est manifeste dans le cas des pionniers, qui défrichent pour que des colons puissent s'installer et cultiver la terre, comme dans le cas des hommes de loi itinérants, qui assurent la sécurité de la vie bourgeoise naissante dans des villes encore marquées par les convois de bétail et le cortège de violences qui les accompagnait - c'est-à-dire traumatisées par une forme sauvage de mobilité. Les héros de l'Ouest se meuvent dans un espace aux limites mobiles, mais par leur mouvement même ils permettent l'émergence d'un espace stable de sédentarité - si bien qu'ils instituent tragiquement un monde dans lequel il n'ont plus de place, car une fois toutes les terres défrichées et toutes les villes pacifiées, l'existence même de ces héros perd son sens : ils deviennent inutiles et sont généralement bannis ou exécutés; de ce point de vue, les héros de l'Ouest américain partagent le destin de ceux que Hegel appelle les «individus historiques", qui est de tomber "comme des douilles vides", une fois leur action accomplie (Hegel 1965, 124). Ici comme ailleurs, le propre des héros est d'agir de telle sorte qu'on n'ait plus besoin de héros. 
10 Leur mobilité, qui leur fait perdre, au terme de leur parcours, toute place dans le monde sédentaire, vient en définitive de ce qu'ils sont radicalement situés entre deux mondes (c'est-à-dire dans aucun des deux), en un sens à la fois temporel et spatial: entre le monde sauvage et le monde civil, entre le monde d'avant et le monde d'après en cela ils sont précisément des hommes de la Frontière. Les héros des guerres indiennes sont souvent décrits par les récits mythiques comme entretenant une relation ambiguë avec leurs adversaires, marquée à la fois par l'hostilité résolue et par une compréhension intime ${ }^{4}$ : c'est ainsi que sont dépeints Kit Carson ou le Général Custer, par exemple, et c'est aussi ce qui caractérise le personnage d'Ethan, joué par John Wayne, dans La Prisonnière du désert de John Ford ${ }^{5}$. De même, les héros de l'ordre comme Wyatt Earp devaient, pour être à la hauteur de leurs adversaires violents, pour être capables de lutter sur leur terrain et avec leurs armes, les connaître intimement et leur ressembler en bien des points (ce que soulignent, grâce à des parallélismes appuyés, des films comme Règlement de comptes à O.K. Corral ${ }^{6}$ ou L'Homme aux colts d'or ${ }^{7}$, adaptés de la légende de Wyatt Earp et de Doc Holliday) ; par leur mode de vie (par leur violence et leur mobilité) ils n'appartiennent donc pas à l'ordre légal et bourgeois qu'ils instaurent, et par leur action en faveur de la loi ils deviennent étrangers au monde sauvage qui était le leur.

\section{Signification politique de cette culture du récit}

11 Ainsi, dans ces légendes, la fondation de la communauté apparaît non pas comme le résultat de la concordance des volontés individuelles autour d'un contrat commun, ni comme un don divin, ni même comme l'œuvre d'un individu exceptionnel qui bâtirait le corps politique et lui donnerait sa loi, mais plutôt comme un processus qui est rendu possible grâce à l'action violente et toute négative d'un homme qui est et demeurera étranger à la communauté fondée; le défricheur ou le marshal n'est ni prince ni législateur ${ }^{8}$, il se contente d'écarter le danger qui jusque là empêchait la société de s'instituer (la nature inhospitalière, l'Indien, ou le cowboy violent étant les trois grandes figures du danger à l'Ouest); son action décisive n'est pas une fabrication de la cité, mais consiste seulement à marquer l'espace où il est possible de fonder une cité, et à quitter cet espace une fois qu'il a été délimité. La dimension narrative des mythes de l'Ouest est précisément ce qui nous révèle ce caractère non démiurgique de la fondation - une institution divine, l'énonciation d'une loi nouvelle ou la conclusion d'un accord juridique ne sont en effet pas des choses qui pourraient être racontées comme on raconte l'histoire de la fondation de l'ordre politique sur la Frontière.

Pourquoi peut-on dire d'une telle représentation de la fondation qu'elle est déterminante pour la construction de la "conscience de soi" politique de la nation américaine - pour la mise en scène de son identité - et pour l'élaboration de son rapport à l'action à venir - pour sa disposition politique ? Tout d'abord, malgré la grande diversité des interprétations de cette légende de l'Ouest (plus ou moins nostalgiques, plus ou moins progressistes ou populistes ${ }^{9}$ ) et malgré la diversité du type de scènes qu'elle offre (guerre contre les Indiens, chasse, colonisation, poursuite des gangs de cowboys, qui chacune évoque un aspect spécifique de la fondation et instaure un régime propre de représentation politique), le trait majeur de cette légende est de faire reposer la fondation sur une action humaine et violente : cette action est accomplie par des individus que le récit distingue, mais elle ne prend son sens qu'au sein de la 
pluralité d'un peuple qui la voit, la commente, l'accompagne, la prolonge, et constitue pour elle une chambre d'écho indispensable. De cette action on peut donc dire, premièrement, qu'elle possède à la fois une dimension individuelle et une dimension collective, deuxièmement qu'elle est ce par quoi la violence peut mettre un terme à la violence et, enfin, qu'elle est ce dont le propre est de produire des histoires plutôt que des choses ${ }^{10}$; et placer ce genre d'action à la racine de l'ordre civil n'est pas sans conséquences pour l'idée qu'on se fait de la nature même du politique.

13 La première thèse sous-jacente à cette représentation est la négation de l'idée d'une autosuffisance du politique : l'ordre politique se fonde sur son contraire, ou plutôt sur sa marge individuelle et violente, et il se régénère à sa frontière ; la fixité s'institue par la mobilité. Se représenter ainsi l'existence de la communauté politique comme conditionnée par son autre et son dehors ancre dans le rapport à soi du groupe la conscience de sa précarité et de la fragilité de toute institution. Et la conséquence pratique de cette représentation est alors une disposition à retrouver l'esprit de la fondation dans une certaine violence ${ }^{11}$, dans une action qui se joue sur les frontières.

L'autre thèse, plus implicite, qui sous-tend cette image de la fondation porte elle aussi sur les conditions prépolitiques du politique, mais insiste sur la nécessité de la légende violente elle-même pour la fondation. L'action individuelle est certes indispensable, mais c'est seulement le fait qu'elle prend la forme de l'épopée, du récit légendaire qui circule dans la communauté, qui lui permettra de produire son effet spécifiquement fondateur; c'est en tant que héros de légende que l'homme de l'Ouest peut établir l'ordre civil dans la prairie, plutôt que par la seule vertu de ses actes. Ce sont en effet les récits des exploits des défricheurs qui suscitent l'enthousiasme pour la conquête de l'Ouest, et ce sont les chansons et les brochures vantant les prouesses au colt des shérifs et des marshals qui assurent d'abord à la loi son aura ; dans ce dernier cas, la contreviolence qui se donne pour but d'être convertie en puissance d'institution a besoin d'être accompagnée du discours légendaire qui la chante pour être lue comme fondatrice d'un ordre politique, et non pas comme simple prolongation du cycle des violences. On peut alors interpréter l'ensemble des productions épiques appartenant aux traditions de l'Ouest américain comme un facteur déterminant de l'institution effective de l'ordre civil dans les anciennes terres sauvages. La constitution de genres artistiques liés à l'histoire de la Frontière (genres musicaux, littéraires, puis cinématographiques) a en elle-même une signification politique, une fonction: accompagner le mouvement par lequel la société américaine s'institue.

Les films westerns les plus élaborés, ceux qui, tout en étant fidèles aux standards du genre, effectuent en leur sein même une réflexion sur ce que fait le genre, mettent en scène cette mise en scène, exposent le processus de mise en légende, et en montrent la nécessité pour que la fondation soit réalisée. C'est le cas de L'homme aux colts d'or de Dmytryck, où une bourgade qui n'a pas encore de statut juridique ne trouve pas d'autre solution, pour faire respecter un semblant de loi dans ses rues, que de recourir aux services tarifés d'un marshal free-lance dont la première force réside dans sa réputation, c'est-à-dire dans la légende violente sédimentée autour de son nom, ce qui a pour effet de rassurer les bourgeois et d'intimider les bandits - et qui va effectivement permettre à la petite ville de passer du règne des cowboys à celui des marchands. Et c'est surtout le cas de L'Homme qui tua Liberty Valance, de John Ford, dont la réplique la plus célèbre est prononcée par un journaliste à qui un important homme politique de l'ancienne Frontière vient de révéler le mensonge sur lequel s'est construite sa propre réputation ; 
il déchire les notes qu'il a prises pendant l'entretien et répond: «This is the West, Sir. When the legend becomes fact, print the legend ! $\gg^{12}$. La construction même de cette phrase traduit la conjonction entre trois éléments: la spécificité de la situation (l'Ouest), la manière dont la déformation des événements, qui permet la fondation (ici, la mythification consistant à raconter que l'homme politique en question a héroïquement défié et vaincu en duel le plus dangereux bandit du territoire), devient le fait lui-même dans la tradition (c'est-à-dire comment le personnage joué par James Stewart devient "l'homme qui a tué Liberty Valance ») et la nécessité de prolonger, dans la façon dont on va imprimer l'histoire dans la presse, le mensonge originel, sous peine de faire s'écrouler l'édifice qui avait été bâti sur ce mensonge, et qui n'est rien de moins que la civilisation, que la transformation du désert en jardin. Ces trois éléments, pris ensemble, font toute l'équivocité du film qui, en montrant à la fois la légende et la mise en légende, ne se réduit ni à la poursuite de la mystification constitutive du genre western, ni à sa pure et simple démystification, mais instaure un régime de lucidité dans lequel on ne peut ignorer ni le prix moral de la fondation de l'ordre politique, ni la nécessité de payer ce prix.

Mais il faut ici s'arrêter sur une autre réplique, moins souvent commentée, et qui révèle d'un autre point de vue ce caractère vital de la légende : dans le flashback qui occupe la majeure partie du film, lorsque, des années plus tôt, le personnage joué par John Wayne apprend à celui qui est interprété par James Stewart qu'il n'est en fait pas l'homme qui a tué Liberty Valance, et qu'il lui enjoint d'endosser malgré cela ce rôle usurpé, il lui rappelle qu'il avait, quelque temps auparavant, appris à lire et à écrire à la femme qu'ils aiment tous les deux : "You taught her how to read and write: now give her something to read and write about! $»^{13}$. C'est précisément cela qu'est, d'après l'étymologie, la légende: ce qui est à lire; et l'institution sociale de l'école, l'existence d'une tradition, d'une transmission écrite, ne prennent leur sens qu'à condition qu'il y ait effectivement une matière narrative à partager, autrement dit une légende qui concerne un public en lui donnant un monde commun, voire qui le constitue par le partage de cette référence commune à des personnages et à des actions.

Et la mise en scène de l'identité américaine se signale justement par son recours fréquent au story-telling, à la narrativité, plutôt qu'à l'image fixe, au symbole, ou à la définition d'une identité de sang ou de terre. Il semble que l'on puisse mettre cette tendance en relation avec la constitution, dans la culture américaine, et particulièrement au cinéma, de grands genres narratifs, notamment le polar, le western et le film de guerre, qui ont un intérêt politique non seulement en vertu de leur objet (la défense de la société contre le crime, le combat de la civilisation contre le monde sauvage, la défense de la patrie ou de la liberté) mais aussi en vertu de leur réception populaire massive; autrement dit ces grands genres sont les relais privilégiés de la représentation que le peuple américain se fait de ce qu'il est et de ce qu'il fait. Et que cette représentation soit avant tout narrative révèle l'importance de l'action dans la définition de cette communauté politique - par différence avec une conception identitaire de la nation ou avec une représentation exclusivement abstraite et juridique de l'ordre politique, qui ne passent pas par la médiation de récits d'action, et avec lesquelles les légendes se trouvent en situation de concurrence.

Cependant, cette narration épique des histoires de la Frontière d'une part n'est pas uniforme (les épisodes en sont variés et peuvent être mobilisés à des fins très diverses ${ }^{14}$ ) et d'autre part n'est pas le seul registre dans lequel les Etats-Unis expriment 
leur rapport à eux-mêmes (ils définissent aussi leur identité politique en référence à des hommes de l'Est ou à des textes fondateurs qui ne sont pas intrinsèquement liés à l'aventure de la Frontière). Il semble clair, en tous cas à la lecture de la trilogie que Richard Slotkin a consacrée à la postérité et aux usages du mythe de la Frontière du XVII à la fin du XX ${ }^{\text {e }}$ siècle $(1973,1985$ et 1992), que l'importance et la tonalité des reprises et des commémorations de la conquête de l'Ouest dans la culture et dans la politique américaines dépendent essentiellement de la qualité de la conjoncture où elles apparaissent; autrement dit, et c'est là que se situe l'enjeu de cette légende du point de vue de la philosophie politique, l'histoire de l'Ouest serait une sorte de miroir que l'Amérique se tendrait à elle-même dans certaines circonstances, en mettant l'accent sur les aspects de la conquête qui l'intéressent au présent, et afin de produire un effet déterminé sur les représentations que les Américains se font d'eux-mêmes, de leur espace et de leur capacité à agir. Ces conjonctures critiques particulières qui, par les réinterprétations polémiques qu'elles suscitent, redonnent vie au mythe de la Frontière ont été analysées par Slotkin, et ce n'est pas ici le lieu de les étudier pour elles-mêmes ; en revanche, le sens général de cette démarche par laquelle une nation se réfère à un aspect essentiel de sa fondation par le biais d'une telle légende doit encore être interrogé.

19 Comme cette légende évoque la fondation de la communauté par ses marges spatiales et sociales, sa remémoration crée de fait un décentrement et une ouverture dans la conception de ce qu'est le groupe politique américain, qui n'apparaît alors ni réductible à ses institutions, ni souverain dans sa définition de lui-même. La légende de la Frontière instaurerait ainsi une forme de lucidité sur la précarité du politique et inviterait à sortir des cadres institutionnels établis pour en défendre l'existence, ce qui explique qu'une telle représentation rencontre un écho plus vif chaque fois que la communauté semble menacée. Ce que produit cette conscience de soi, ce n'est alors pas un repli sur une identité ethnique ou sur un espace acquis, mais une incitation à l'action, à la refondation par l'action, qui définit un rapport spécifique à l'espace s'appuyant sur les propriétés spatiales de l'Ouest. On l'a vu, l'Ouest est un espace mobile, sans cesse réorganisé par des hommes itinérants et par les récits, qui euxmêmes circulent, des actions de ces hommes; mais, plus radicalement, l'Ouest est un lieu où l'on va, comme le dit la formule synthétique popularisée par Horace Greeley "Go west, young man, and grow up with the country!"; ce n'est pas un espace où l'on peut être immobile, car il est défini en tant que tel par la mobilité de ses héros. Non seulement ce n'est pas un espace qui correspond à une délimitation géographique précise, mais c'est un espace mythique dont le propre est d'être le lieu vers lequel le mouvement s'oriente (s'il est permis de parler ici d'orientation) ; en d'autres termes il n'y a pas de West sans « Go west ». La frontière de l'Ouest est donc un espace investi par l'imaginaire politique comme l'espace de l'expansion coloniale et de la mobilité par excellence, comme étant ce mouvement lui-même, et son évocation dans la légende intervient dans des conjonctures où la communauté ressent de nouveau ce besoin d'air, d'espace ouvert aux possibilités indéfinies, qui se trouve par principe aux marges, à l'extérieur de l'espace politique institutionnel ${ }^{15}$.

20 A partir du moment où naît le sentiment que la Frontière s'est refermée, lors donc que l'Ouest n'est plus le lieu mobile et indéterminé vers lequel tout mouvement se dirige, c'est le thème de la «nouvelle frontière " qui, dès avant Kennedy et après lui, commande les relectures conflictuelles de la première frontière dans les grands moments de refondation, comme la sortie de la Grande Dépression, la lutte pour les 
Droits civiques, la conquête spatiale, entre autres... Dans ces rhétoriques de la nouvelle frontière, la référence à la Frontière n'a pas nécessairement de signification spatiale, mais elle constitue cet espace-mouvement qui permet de penser et de produire les conditions d'une action politique qui soit à nouveau fondatrice, qui régénère la nation et lui offre des possibilités qui excèdent le champ clos du territoire concret des ÉtatsUnis et de leurs institutions.

\section{BIBLIOGRAPHIE}

Arendt, Hannah. Condition de l'homme moderne. Paris: Pocket Agora, 2002.

Barthes, Roland. « Le mythe, aujourd'hui. » In Mythologies. Paris : Le Seuil, Points, 1970. 179-233.

Bayertz, Kurt. «Zur Ästhetik des Western ». In Zeitschrifft für Allgemeine Kunstwissenschaft. Eds. Josef Frucht and Maria Moog-Grunewald. Hamburg: Felix Meiner Ferlag, 2003. 69-82.

---. « Hegel und der wilde Westen ». In Hegel-Perspektiven seiner Philosophie heute. Ed. Bernhard Heidtmann. Köln : Pahl-Rugenstein, 1981. 131-48.

Bazin, André. « Le Western ou le cinéma américain par excellence ». In Qu'est-ce que le cinéma? Paris : Cerf, 1961. 217-228.

Gérard, Valérie. « Politique et violence selon Hannah Arendt. » In Violences. Anthropologie, politique, philosophie. Ed. Guillaume Sibertin-Blanc. Paris : Europhilosophie Éditions, «Bibliothèque de philosophie sociale et politique », 2010. 29-53. Disponible en ligne : http://www.europhilosophie-editions.eu/fr/spip.php?article40

Hegel, G. W. F. Esthétique. Quatrième partie, « La poésie » Paris : Flammarion, « Champs », 1979.

---. La Raison dans l'Histoire. Paris : 10-18, Plon, 1965.

Pippin, Robert B. « What Is a Western? Politics and Self-Knowledge in John Ford's The Searchers » In Critical Inquiry 35 (Winter 2009). 223-253. Disponible en ligne :

https://webshare.uchicago.edu/users/rbp1/Public/Searchers.pdf ?uniq =p1tndg

Repris dans: Pippin, Robert B. Hollywood Westerns and American Myth: the Importance of Howard Hawks and John Ford for Political Philosophy. New Haven: Yale University Press, 2010.

Slotkin, Richard. Regeneration through Violence: the Mythology of the American Frontier, 1600-1860. Middletown: Wesleyan University Press, 1973.

---. The Fatal Environment the Myth of the Frontier in the Age of Industrialization. 1800-1890. New York: Atheneum, 1985.

---. Gunfighter Nation: the Myth of the Frontier in Twentieth-Century America. New-York: Atheneum, 1992. 


\section{NOTES}

1. "The Greeks have the Iliad; the Jews, the Hebrew Bible; the Romans, the Aeneid; the Germans, the Nibelungenlied; the Scandinavians, the Njáls saga; the Spanish have the Cid; the British have the Arthurian legends. The Americans have John Ford » (Pippin 224, je traduis). Dans ce passage, Robert Pippin renvoie aux textes de Bayertz (1981 et 2003), ainsi qu'à Bazin (1961).

2. En partant des critères hégéliens de définition de l'épopée, on pourrait même considérer que les légendes classiques de l'Ouest, qui apparaissent pour ainsi dire à même la Frontière, sont davantage conformes au concept de l'épique que ne l'est, par exemple, l'Énéide de Virgile, qui est une composition poétique tardive, et non pas un récit authentiquement fondateur circulant depuis les origines dans la culture populaire. L'absence de distance et le sérieux dans le traitement du sujet sont deux conditions indispensables à la production du genre épique qui étaient réunies dans l'âge classique du Western, mais qui furent perdues dès lors que l'on cessa de croire à la puissance fondatrice de l'aventure de la Frontière-et c'est justement à ce moment-là que le genre perdit son caractère proprement national.

3. De nombreux films westerns montrent des héros entrant dans une ville précédés des récits de leurs exploits et insistent ainsi sur l'importance de leur réputation (qui concerne en général l'habileté au colt) pour l'accomplissement de leur action. Il en sera question dans la dernière partie de cet article.

4. «L'homme-qui-connaît-les-Indiens » (« the man who knows Indians ») est précisément l'une des figures majeures héritées du mythe de la Frontière, dont Slotkin retrace le destin dans Gunfighter Nation (1992).

5. La Prisonnière du désert ; titre original : The Searchers, réalisé par John Ford (1956).

6. Règlement de comptes à O.K. Corral; titre original : Gunfight at the O.K. Corral, réalisé par John Sturges (1957).

7. L'Homme aux colts d'or ; titre original : Warlock, réalisé par Edward Dmytryck (1959) et tiré du roman d'Oakley Hall (Warlock, 1958) qui, en changeant les noms des lieux et des personnages, revisitait très directement la légende de Tombstone, et le célèbre gunfight opposant le marshal et le clan des bandits.

8. S'il arrive parfois à certains héros de proclamer «Je serai la loi » ("I'll be the law»), comme le personnage joué par Henry Fonda dans L'Homme aux colts d'or (op. cit., 16'12), il ne s'agit en aucun cas d'une prétention à donner une loi durable à la communauté, à fonder souverainement son ordre légal à venir, mais « seulement » de décréter que tant que sa mission n'est pas terminée, la ville ne connaîtra d'autre loi que les décrets qu'il prendra et fera appliquer lui-même.

9. Nous reprenons ici les catégories qu'utilise Richard Slotkin (1992, 29-62) pour analyser notamment les différences entre la lecture de la Frontière par Frederick Jackson Turner et celle qu'en fait Theodore Roosevelt.

10. Cette analyse des liens entre l'action et le récit s'inspire principalement du chapitre $\mathrm{V}$ de Condition de l'homme moderne, de Hannah Arendt. Pour une discussion des rapports ambivalents entre l'action politique et la violence, voir "Politique et violence selon Hannah Arendt", par Valérie Gérard, art. cité en bibliographie. 
11. C'est à partir de cette disposition que $R$. Slotkin forge le concept de "régénération par la violence" («regeneration through violence»), qui donne son titre au premier volume de sa trilogie de la Frontière (Slotkin 1973).

12. "C'est l'Ouest, ici, Monsieur. Quand la légende devient le fait, imprimez la légende !" (je traduis). In L'Homme qui tua Liberty Valance; titre original: The Man who shot Liberty Valance, réalisé par John Ford (1962), 1h54'50» .

13. « Tu lui as appris à lire et à écrire : maintenant, donne-lui quelque chose à lire et à écrire !" (je traduis). In L'Homme qui tua Liberty Valance, 1h53'36 ».

14. La dimension raciale de certains des mythes de l'Ouest montre, par exemple, qu'ils peuvent même être mobilisés à des fins littéralement antipolitiques. Mais les réduire à cette seule dimension serait faire peu de cas de ce qu'ils nous disent, par exemple, des rapports du droit et de la force, ou de la politique et de la violence.

15. Chaque fois que ces "marges » seront comprises en un sens purement spatial, on recherchera évidemment la régénération de la communauté politique dans la continuation de l'aventure coloniale, c'est-à-dire dans l'impérialisme. Mais cette lecture n'épuise pas les réinterprétations politiques de l'histoire de la Frontière.

\section{RÉSUMÉS}

La mise en scène mythique de la mobilité des héros de l'Ouest et de toute la société de la Frontière instaure un rapport singulier à l'espace qu'il convient d'élucider si l'on veut essayer de comprendre la représentation que l'Amérique se fait de son identité politique. À cette fin, nous analyserons d'abord le rapport entre le contenu des légendes de l'Ouest et le contexte de leur formation, avant d'étudier la signification politique des représentations de la fondation à l'œuvre dans ces légendes.

The mythic staging of the mobility of western heroes, and of the whole Frontier society, establishes a specific relation with space, a relation whose nature should be defined in order to understand America's vision of its own political identity. In this perspective, we will first analyze the link between the content of western legends and the context of their making, and then study the political significance of the depictions of the founding at work in these legends.

\section{INDEX}

Keywords : philosophy, west, hero, frontier, space, mobility, politics, myth, legend, narrative, epic, violence, cinema, western movies, United States

Mots-clés : philosophie, héros, frontière, espace, mobilité, politique, mythe, légende, récit, épopée, violence, cinéma, western, Ouest américain 


\section{AUTEURS}

\section{DANIEL AGACINSKI}

Doctorant

Université Toulouse 2 - Le Mirail

daniel.agacinski@univ-tlse2.fr 